\title{
Chemical Synthesis of a Very Long-Chain Fatty Acid, Hexacosanoic Acid (C26:0), and the Ceramide Containing Hexacosanoic Acid
}

\author{
Yoshinori Yамамото, Toshimasa Ітон and Keiko Yамамото* \\ Laboratory of Drug Design and Medicinal Chemistry, Showa Pharmaceutical University, \\ 3-3165 Higashi-Tamagawagakuen, Machida, Tokyo 194-8543, Japan
}

(Received December 24, 2014)

\begin{abstract}
Summary Hexacosanoic acid (C26:0) (1), a very long-chain fatty acid, is related to various diseases such as adrenoleukodystrophy (ALD), adrenomyeloneuropathy (AMN) and atherosclerosis. As the level of $\mathbf{1}$ higher than the normal is related to diseases above, hexacosanoic acid (1) and the ceramide $\mathbf{2}$, which contains $\mathbf{1}$, are thought to play an important role in various tissues. Hexacosanoic acid (1) is known to be a waxy solid and to be hard to dissolve in water as well as organic solvents. Due to this physical property, it is not easy to handle hexacosanoic acid (1) in a laboratory. Therefore, efficient chemical synthesis of the compounds $\mathbf{1}$ and $\mathbf{2}$ has not been reported. Here, we report a versatile synthetic method for hexacosanoic acid (1) and the ceramide $\mathbf{2}$ containing the fatty acid $\mathbf{1}$. Synthesis of hexacosanoic acid (1) was achieved by applying the coupling of two alkyl units as a key step. Ceramide $\mathbf{2}$ was efficiently synthesized by applying the reported procedure together with hexacosanoic acid (1) synthesized here. This synthetic strategy has an advantage of getting various carbon chain length fatty acids and their ceramides by using a variety of carbon chain units. This method is also applicable for large-scale synthesis. In addition, these compounds $\mathbf{1}$ and $\mathbf{2}$ are useful for investigation of details of these compounds related to diseases such as ALD and AMN.
\end{abstract}

Key Words hexacosanoic acid, cerotic acid, ceramide, chemical synthesis, adrenoleukodystrophy

Long-chain fatty acids are biogenic substances. They are part of signaling pathways, sources of energy, and constituents of cell membranes. However, the physiological roles of some long-chain fatty acids, especially very long-chain fatty acids (VLCFA) such as hexacosanoic acid $\mathbf{1}$, remain unclear.

Hexacosanoic acid (C26:0) (1), which is also called cerotic acid, is related to adrenoleukodystrophy (ALD) $(1,2)$, adrenomyeloneuropathy (AMN) (1), atherosclerosis (3), coronary artery disease $(4)$, aging $(3,5)$ and metabolic syndrome (6). As the level of $\mathbf{1}$ higher than the normal is related to the diseases above, hexacosanoic acid (1) and the sphingolipid containing $\mathbf{1}$ are thought to play an important role in multiple tissues (5). Hexacosanoic acid is known to be a waxy solid and to be hard to dissolve in water as well as organic solvents. It has been reported that due to this physical property of hexacosanoic acid, its accumulation causes a decrease the fluidity of cell membranes (3). Ceramide, which is a kind of sphingolipid, has a function as a second messenger, so it is suggested that the ceramide is also related to those diseases. However, efficient chemical synthesis of these compounds has not been reported. In this article,

E-mail: yamamoto@ac.shoyaku.ac.jp

Abbreviations: ALD, adrenoleukodystrophy; AMN, adrenomyeloneuropathy; EEDQ, N-ethoxycarbonyl-2-ethoxy-1,2dihydroquinoline; TBAF, tetra-n-butylammonium fluoride; VLCFA, very long-chain fatty acids. we report a useful synthetic method for hexacosanoic acid (1) and ceramide $\mathbf{2}$ containing a hexacosanoic acid (Fig. 1).

\section{MATERIALS AND METHODS}

General experimental procedures. All reagents were purchased from commercial sources and used without further purification. Organic solvents used were dried by standard methods. All reactions were performed under a nitrogen atmosphere. Silica gel (Kanto Chemical Silica gel 60 , Spherical, $63-210$ or $40-50 \mu \mathrm{m}$ ) was used for column chromatography, and pre-coated silica gel $60 \mathrm{~F}_{254}$ plates $\left(0.25 \mathrm{~mm}\right.$, Merck) were used for TLC. ${ }^{1} \mathrm{H}$ NMR (300 MHz) and ${ }^{13} \mathrm{C}$ NMR (75 MHz) were recorded using a Bruker AV300 instrument in $\mathrm{CDCl}_{3}$ solution with TMS or $\mathrm{CDCl}_{3}(\delta 7.26)$ and $\mathrm{CDCl}_{3}(\delta 77.23)$ as an internal standard, respectively. The chemical shifts are given in $\delta$ values. Splitting patterns are indicated as follows: s, singlet; d, doublet; t, triplet; q, quartet; $\mathrm{m}$, multiplet. Mass spectra were recorded on a JEOL AccuTOF LCplus JMS-T100LP and JEOL MS700 spectrometer using NBA as the positive-ion FAB matrix.

1-Bromo-10-(methoxymethoxy)decane (3): To a solution of 10-bromo-1-decanol $(2.01 \mathrm{~g}, 8.47 \mathrm{mmol})$ and $\mathrm{N}, \mathrm{N}$-diisopropylethylamine $(3.08 \mathrm{~g}, 23.9 \mathrm{mmol})$ in $\mathrm{CH}_{2} \mathrm{Cl}_{2}(10 \mathrm{~mL})$ at $0^{\circ} \mathrm{C}$ was added chloromethyl methyl ether $(1.85 \mathrm{~g}, 22.9 \mathrm{mmol})$ and the mixture was stirred at $0^{\circ} \mathrm{C}$ for $3 \mathrm{~h}$. The reaction was quenched with $1 \mathrm{~N}$ $\mathrm{HCl}$ and extracted with $\mathrm{CH}_{2} \mathrm{Cl}_{2}$. The organic layer was 


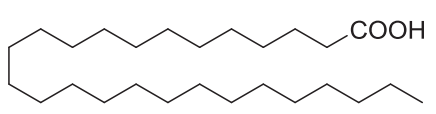

hexacosanoic acid 1

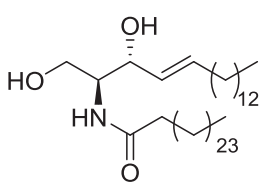

ceramide 2
Fig. 1. Structures of hexacosanoic acid and the ceramide containing hexacosanoic acid.

washed with saturated $\mathrm{NaHCO}_{3}$ aq. and brine, dried over $\mathrm{MgSO}_{4}$, and evaporated. The residue was flash-chromatographed on silica gel (50 g, hexane/AcOEt, and then $80: 1)$ to give compound $3(1.78 \mathrm{~g}, 75 \%) .3{ }^{1} \mathrm{H}$ NMR $\delta$ $1.02-1.51\left(12 \mathrm{H}, \mathrm{m},-\mathrm{CH}_{2^{-}}\right), 1.51-1.70\left(2 \mathrm{H}, \mathrm{m},-\mathrm{CH}_{2^{-}}\right.$ $\left.\mathrm{CH}_{2}-\mathrm{O}-\mathrm{CH}_{2}-\mathrm{O}-\mathrm{CH}_{3}\right), 1.70-1.98\left(2 \mathrm{H}, \mathrm{m}, \mathrm{Br}-\mathrm{CH}_{2}-\mathrm{CH}_{2}{ }^{-}\right)$, $3.36\left(3 \mathrm{H}, \mathrm{s},-\mathrm{O}-\mathrm{CH}_{2}-\mathrm{O}-\mathrm{C}_{3}\right)$ ) $3.41(2 \mathrm{H}, \mathrm{t}, J=6.9 \mathrm{~Hz}$, $\left.\mathrm{Br}-\mathrm{C}_{2} \underline{2}^{-}\right), 3.52\left(2 \mathrm{H}, \mathrm{t}, J=6.6 \mathrm{~Hz},-\mathrm{CH}_{2}-\mathrm{O}-\mathrm{CH}_{2}-\mathrm{O}-\mathrm{CH}_{3}\right)$, $4.62\left(2 \mathrm{H}, \mathrm{s},-\mathrm{O}-\mathrm{C}_{2}-\mathrm{O}-\mathrm{CH}_{3}\right) .{ }^{13} \mathrm{C} \mathrm{NMR} \delta 26.3,28.3$, $28.9,29.49,29.50,29.59,29.9,33.0,34.1,55.2$, 67.9, 96.5. IR (neat): 2928, 2854, 1150, 1111, 1045, $644,563 \mathrm{~cm}^{-1}$.

1-(Methoxymethoxy)hexacosane (4): Hexadecanyl magnesium bromide was prepared from $\mathrm{Mg}$ $(0.954 \mathrm{~g}, 39.2 \mathrm{mmol})$ and 1-bromohexadecane $(8.16 \mathrm{~g}$, $26.7 \mathrm{mmol})$ in THF $(22 \mathrm{~mL})$. To a mixture of $3(0.50 \mathrm{~g}$, $1.79 \mathrm{mmol}), \mathrm{Li}_{2} \mathrm{CuCl}_{4}(100 \mathrm{mM}$ solution in THF, 5.3 $\mathrm{mL})$ and $\mathrm{N}$-methylpyrrolidone $(0.69 \mathrm{~mL}, 7.16 \mathrm{mmol})$ was slowly added hexadecanyl magnesium bromide $(0.476 \mathrm{M}, 7.6 \mathrm{~mL}, 3.62 \mathrm{mmol})$ at room temperature under nitrogen. The deep purple solution was stirred at room temperature for $2.5 \mathrm{~h}$. The reaction was quenched with saturated $\mathrm{NH}_{4} \mathrm{Cl}$ aq. and extracted with AcOEt. The organic layer was washed with water and brine, dried over $\mathrm{MgSO}_{4}$, and evaporated. The residue was flashchromatographed on silica gel $(50 \mathrm{~g}$, hexane/AcOEt, $80: 1)$ to give compound $4(0.623 \mathrm{~g}, 81 \%) . \mathbf{4}^{1} \mathrm{H}$ NMR $\delta 0.88\left(3 \mathrm{H}, \mathrm{t}, J=6.5 \mathrm{~Hz},-\mathrm{CH}_{2}-\mathrm{CH}_{3}\right), 1.14-1.47(46 \mathrm{H}$, m, $\left.-\mathrm{CH}_{2}-\right), 1.57-1.65\left(2 \mathrm{H}, \mathrm{m},-\mathrm{CH}_{2}-\mathrm{CH}_{2}-\mathrm{O}-\mathrm{CH}_{2}-\mathrm{O}-\mathrm{CH}_{3}\right)$, $3.36\left(3 \mathrm{H}, \mathrm{s},-\mathrm{O}-\mathrm{CH}_{2}-\mathrm{O}-\mathrm{C}_{3}\right), 3.52(2 \mathrm{H}, \mathrm{t}, J=6.6 \mathrm{~Hz}$, $\left.-\mathrm{C}_{2}-\mathrm{O}-\mathrm{CH}_{2}-\mathrm{O}-\mathrm{CH}_{3}\right), 4.62\left(2 \mathrm{H}, \mathrm{s},-\mathrm{O}-\mathrm{C}_{2}-\mathrm{O}-\mathrm{CH}_{3}\right) .{ }^{13} \mathrm{C}$ NMR $\delta 14.3,22.9,26.5,29.6,29.7,29.8$ (2 carbons), 29.90, 29.93 (14 carbons), 30.0, 32.2, 55.3, 68.1, 96.6. MS $\left(\mathrm{FAB}^{+}\right) \mathrm{m} / \mathrm{z}$ (relative intensity): $426\left(\mathrm{M}^{+}, 4\right)$, 395 (8), 307 (76), 289 (49), 154 (100), 136 (56). IR $\left(\mathrm{CHCl}_{3}\right): 2926,2854,1614,1605,947 \mathrm{~cm}^{-1}$.

Hexacosan-1-ol (5): A solution of MOM ether 4 $(0.201 \mathrm{~g}, 0.471 \mathrm{mmol})$ in $3 \% \mathrm{HCl}-\mathrm{THF}(20 \mathrm{~mL})$ was stirred at $50^{\circ} \mathrm{C}$ for $4 \mathrm{~h}$. The mixture was evaporated to give crude compound 5 (0.177 g, 98\%). $5{ }^{1} \mathrm{H}$ NMR $\delta$ $0.88\left(3 \mathrm{H}, \mathrm{t}, \mathrm{J}=6.8 \mathrm{~Hz},-\mathrm{CH}_{2}-\mathrm{CH}_{3}\right), 1.13-1.42(46 \mathrm{H}, \mathrm{m}$, $\left.-\underline{\mathrm{C}}_{2} \underline{2}^{-}\right), 1.52$ (2 H, s, - $\left.\underline{\mathrm{CH}}_{2}-\mathrm{CH}_{2}-\mathrm{OH}\right), 3.56-3.68$ (2 H, m, $\left.-\mathrm{CH}_{2}-\mathrm{CH}_{2}-\mathrm{OH}\right) .{ }^{13} \mathrm{C}$ NMR $\delta 14.4,22.9,26.0,29.6,29.7$, 29.8 (2 carbons), 29.93 (14 carbons), 29.98, 32.2, 33.1, 63.4.

Hexacosanal (6): A mixture of alcohol 5 (0.20 g, $0.523 \mathrm{mmol})$, Dess-Martin periodinane (15 wt\% in $\mathrm{CH}_{2} \mathrm{Cl}_{2}$ solution, $\left.2.7 \mathrm{~mL}, 0.785 \mathrm{mmol}\right)$ and $\mathrm{NaHCO}_{3}$ $(0.836 \mathrm{~g}, 9.951 \mathrm{mmol})$ in $\mathrm{CH}_{2} \mathrm{Cl}_{2}(67 \mathrm{~mL})$ was warmed to $30^{\circ} \mathrm{C}$ to dissolve compound $\mathbf{5}$ in the solution and then the solution was stirred at $30^{\circ} \mathrm{C}$ for $2 \mathrm{~h}$. After the addition of saturated $\mathrm{NaHCO}_{3}$ aq. and saturated $\mathrm{Na}_{2} \mathrm{~S}_{2} \mathrm{O}_{3}$ aq., the mixture was extracted with $\mathrm{CH}_{2} \mathrm{Cl}_{2}$. The organic layer was washed with water and brine, dried over $\mathrm{MgSO}_{4}$, and evaporated. The residue was chromatographed on silica gel $\left(20 \mathrm{~g}, \mathrm{CH}_{2} \mathrm{Cl}_{2} /\right.$ hexane, $1: 30$ and then $\left.\mathrm{CH}_{2} \mathrm{Cl}_{2}\right)$ to give compound $6(0.1679 \mathrm{~g}, 84 \%) .6{ }^{1} \mathrm{H}$ NMR $\delta 0.88\left(3 \mathrm{H}, \mathrm{t}, J=6.7 \mathrm{~Hz},-\mathrm{CH}_{2}-\mathrm{CH}_{3}\right), 1.16-1.38$ (44 H, m, $\left.-\underline{\mathrm{CH}}_{2}-\right)$, 1.59-1.70 (2 H, m, $\left.-\underline{\mathrm{CH}}_{2}-\mathrm{CH}_{2}-\mathrm{CHO}\right)$, $2.42\left(2 \mathrm{H}, \mathrm{dt}, J=7.23,1.85 \mathrm{~Hz},-\mathrm{CH}_{2}-\mathrm{CHO}\right), 9.77(1 \mathrm{H}$, t, $1.86 \mathrm{~Hz},-\mathrm{CHO}) .{ }^{13} \mathrm{C}$ NMR $\delta 14.3,22.3,22.9,29.4$, 29.6, 29.7, 29.81, 29.89, 29.93 (15 carbons), 32.2,

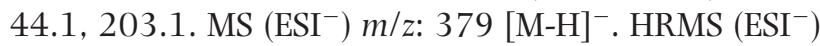
calcd. for ${ }^{12} \mathrm{C}_{26}{ }^{1} \mathrm{H}_{51}{ }^{16} \mathrm{O}_{1} 379.3940$, found 379.3947 . IR $\left(\mathrm{CHCl}_{3}\right): 2926,2854,1724 \mathrm{~cm}^{-1}$.

Hexacosanoic acid (1): To a solution of aldehyde $6(0.0999 \mathrm{~g}, 0.262 \mathrm{mmol})$ and 2-methyl-2-butene (1.3 mL, $12.3 \mathrm{mmol})$ in tert-butyl alcohol $(51 \mathrm{~mL})$ was added a solution of sodium chlorite $(0.22 \mathrm{~g}, 2.43 \mathrm{mmol})$ and sodium dihydrogenphosphate dihydrate $(0.29 \mathrm{~g}$, $1.82 \mathrm{mmol})$ in water $(2.1 \mathrm{~mL})$ dropwise over $4 \mathrm{~min}$. The mixture was stirred at room temperature for $1 \mathrm{~h}$. After volatile components were removed under vacuum, $2 \mathrm{~N} \mathrm{HCl}$ was added. The mixture was extracted with $\mathrm{CH}_{2} \mathrm{Cl}_{2}$. The organic layer was washed with water and brine, dried over $\mathrm{MgSO}_{4}$, and evaporated. The residue was chromatographed on silica gel $\left(1 \mathrm{~g}, \mathrm{MeOH} / \mathrm{CH}_{2} \mathrm{Cl}_{2}\right.$, $1: 15)$ to give compound $\mathbf{1}(0.0772 \mathrm{~g}, 74 \%) . \quad{ }^{1} \mathrm{H}$ NMR $\delta 0.88\left(3 \mathrm{H}, \mathrm{t}, J=6.72 \mathrm{~Hz},-\mathrm{CH}_{2}-\mathrm{CH}_{3}\right), 1.14-1.40$ (44 H, m, - $\underline{\mathrm{CH}}_{2^{-}}$), 1.61-1.69 (2 H, m, $\left.-\underline{\mathrm{CH}}_{2}-\mathrm{CH}_{2}-\mathrm{CHO}\right)$, $2.36\left(2 \mathrm{H}, \mathrm{t}, J=7.5 \mathrm{~Hz},-\mathrm{CH}_{2}-\mathrm{COOH}\right) .{ }^{13} \mathrm{C} \mathrm{NMR} \delta 14.4$, $22.9,24.9,29.3,29.5,29.6,29.7,29.8,29.9$ (15 carbons), 32.2, 33.8, 177.8. MS (ESI $\left.{ }^{-}\right) \mathrm{m} / \mathrm{z}: 395[\mathrm{M}-\mathrm{H}]^{-}$. HRMS (ESI ${ }^{-}$) calcd. for ${ }^{12} \mathrm{C}_{26}{ }^{1} \mathrm{H}_{51}{ }^{16} \mathrm{O}_{2} 395.3889$, found 395.3903. IR $\left(\mathrm{CHCl}_{3}\right)$ : 2926, 2854, $1724 \mathrm{~cm}^{-1}$.

(E)-hexadec-2-enal (8): To a solution of oxalyl chloride $(3.1 \mathrm{~mL}, \quad 36.1 \mathrm{mmol})$ in $\mathrm{CH}_{2} \mathrm{Cl}_{2}(160 \mathrm{~mL})$ was added a solution of DMSO $(5.1 \mathrm{~mL}, 71.9 \mathrm{mmol})$ in $\mathrm{CH}_{2} \mathrm{Cl}_{2}(20 \mathrm{~mL})$ at $-78^{\circ} \mathrm{C}$. After being stirred for $10 \mathrm{~min}$, a solution of alcohol $7(7.19 \mathrm{~g}, 29.9 \mathrm{mmol})$ in $\mathrm{CH}_{2} \mathrm{Cl}_{2}(83 \mathrm{~mL})$ was added and the mixture was stirred at $-78^{\circ} \mathrm{C}$ for $15 \mathrm{~min}$. $\mathrm{Et}_{3} \mathrm{~N}(21 \mathrm{~m} \mathrm{~L}, 150.7 \mathrm{mmol})$ was added to the reaction mixture and stirred for $30 \mathrm{~min}$ at $-78^{\circ} \mathrm{C}$. Then the mixture was allowed to warm to room temperature. The reaction was quenched with water, and the mixture was extracted with $\mathrm{CH}_{2} \mathrm{Cl}_{2}$. The organic layer was washed with brine, dried over $\mathrm{MgSO}_{4}$, and evaporated. The residue was chromatographed on silica gel (40 g, hexane/AcOEt, $20: 1$ ) to give compound 8 (6.93 g, 97\%). ${ }^{1} \mathrm{H}$ NMR $\delta 0.88\left(3 \mathrm{H}, \mathrm{t}, J=6.7 \mathrm{~Hz},-\mathrm{CH}_{2}-\right.$ $\left.\mathrm{C}_{3}{ }_{3}\right), 1.12-1.41\left(20 \mathrm{H}, \mathrm{m},-\underline{\mathrm{CH}}_{2^{-}}\right), 1.41-1.55(2 \mathrm{H}, \mathrm{m}$, $\left.-\mathrm{CH}=\mathrm{CH}-\mathrm{CH}_{2}-\mathrm{CH}_{2^{-}}\right), 2.27-2.38\left(2 \mathrm{H}, \mathrm{m},-\mathrm{CH}=\mathrm{CH}-\underline{\mathrm{H}}_{2^{-}}\right)$, 6.11 (1 H, ddt, $J=15.6,7.9,1.5 \mathrm{~Hz}, \mathrm{OHC}-\mathrm{CH}=\mathrm{CH}-$ ), 6.85 ( $1 \mathrm{H}, \mathrm{dt}, J=15.6,6.8 \mathrm{~Hz}, \mathrm{OHC}-\mathrm{CH}=\mathrm{CH}-), 9.50$ (1 $\mathrm{H}, \mathrm{d}, \mathrm{J}=7.9 \mathrm{~Hz}, \mathrm{OHC}-)$.

(S)-3-(2-Bromoacetyl)-4-isopropyloxazolidin2-one (9): To a solution of $(S)$-4-isopropyl-2-oxazolidinone $(1.29 \mathrm{~g}, 10.0 \mathrm{~mol})$ in THF $(50 \mathrm{~mL})$ at $-78^{\circ} \mathrm{C}$ was added $n$-BuLi (1.65 $\mathrm{M}$ in hexanes, $6.1 \mathrm{~mL}, 10.1 \mathrm{mmol}$ ). The mixture was stirred for $30 \mathrm{~min}$ at $-20^{\circ} \mathrm{C}$ and 
then cooled to $-78^{\circ} \mathrm{C}$. Bromoacetyl bromide $(0.9 \mathrm{~mL}$, $10.4 \mathrm{mmol})$ in THF $(15 \mathrm{~mL})$ was added to the solution and the mixture was allowed to warm slowly to room temperature. The reaction was quenched with phosphate buffer ( $\mathrm{pH} 7$ ), and the mixture was extracted with AcOEt. The organic layer was washed with water and brine, dried over $\mathrm{MgSO}_{4}$, and evaporated. The residue was chromatographed on silica gel (40 g, hexane/ AcOEt, $5: 1)$ to give compound 9 (1.72 g, 69\%). $9{ }^{1} \mathrm{H}$ NMR $\delta 0.91\left(3 \mathrm{H}, \mathrm{d}, J=6.9 \mathrm{~Hz},-\underline{\mathrm{C}}_{3}\right), 0.94(3 \mathrm{H}, \mathrm{d}$, $\left.J=7.5 \mathrm{~Hz},-\underline{\mathrm{CH}}_{3}\right), 2.34-2.51\left(1 \mathrm{H}, \mathrm{m},-\mathrm{C} \underline{\mathrm{H}}\left(\mathrm{CH}_{3}\right)_{2}\right), 4.24-$ 4.64 (5 H, m, - $-\mathrm{C} \underline{\mathrm{H}}_{2}-\mathrm{Br},-\mathrm{O}-\mathrm{C}_{2}-\underline{\mathrm{H}}_{2}-$ ).

(S)-3-((2S,3R,E)-2-Bromo-3-hydroxyoctadec4-enoyl)-4-isopropyloxazolidin-2-one (10): To a stirred solution of bromoacetyl $9(8.41 \mathrm{~g}, 33.6 \mathrm{mmol})$ in ether $(115 \mathrm{~mL})$ at $-78^{\circ} \mathrm{C}$ was added $\mathrm{Et}_{3} \mathrm{~N}(6.4 \mathrm{~mL}$, $45.9 \mathrm{mmol})$. After $5 \mathrm{~min}$, dibutylborontriflate $(1.0 \mathrm{M}$ in $\mathrm{CH}_{2} \mathrm{Cl}_{2}, 33.6 \mathrm{~mL}$ ) was added slowly. After $15 \mathrm{~min}$, the mixture was warmed to room temperature and stirred for $2 \mathrm{~h}$. The mixture was slowly cooled to $-78^{\circ} \mathrm{C}$ again and a solution of aldehyde $\mathbf{8}(5.03 \mathrm{~g}, 21.1 \mathrm{mmol})$ in $\mathrm{Et}_{2} \mathrm{O}(100 \mathrm{~mL})$ was added. The mixture was stirred for $45 \mathrm{~min}$ at $-78^{\circ} \mathrm{C}$, and then for $1.5 \mathrm{~h}$ at $0^{\circ} \mathrm{C}$. The reaction mixture was diluted with ether and poured into saturated $\mathrm{NaHSO}_{4}$ aq. The organic layer was washed with saturated $\mathrm{NaHSO}_{4}$ aq. and brine and then evaporated. The residue was dissolved in $\mathrm{Et}_{2} \mathrm{O}$ and cooled to $0{ }^{\circ} \mathrm{C}$. To the solution was slowly added a $1: 1$ mixture of $\mathrm{MeOH}$ and $30 \%$ aq. $\mathrm{H}_{2} \mathrm{O}_{2}(150 \mathrm{~mL})$. The mixture was stirred for $1 \mathrm{~h}$ at $0^{\circ} \mathrm{C}$ and diluted with $\mathrm{Et}_{2} \mathrm{O}$. The mixture was poured into saturated $\mathrm{NaHCO}_{3}$ aq. and extracted with $\mathrm{Et}_{2} \mathrm{O}$. The organic layer was washed with saturated $\mathrm{NaHCO}_{3}$ aq. and brine, dried over $\mathrm{MgSO}_{4}$, and evaporated. The residue was flash-chromatographed on silica gel (200 g, hexane/AcOEt, $4: 1)$ to give compound 10 (7.87 g, 76\%). $10{ }^{1} \mathrm{H}$ NMR $\delta 0.88(3 \mathrm{H}, \mathrm{t}, J=6.6 \mathrm{~Hz}$, $\left.-\mathrm{CH}_{3}\right), 0.94\left(6 \mathrm{H}, \mathrm{d}, J=7.2 \mathrm{~Hz},-\mathrm{CH}\left(\mathrm{CH}_{3}\right)_{2}\right), 1.11-1.43$ $\left(22 \mathrm{H}, \mathrm{m},-\mathrm{CH}_{2^{-}}\right), 1.99-2.09$ (2 H, m, - $\left.\mathrm{CH}=\mathrm{CH}-\underline{\mathrm{C}}_{2}-\right)$, 2.30-2.50 (1 H, m, - $\left.\underline{\mathrm{H}}\left(\mathrm{CH}_{3}\right)_{2}\right), 3.11(1 \mathrm{H}, \mathrm{d}, \mathrm{J}=2.9 \mathrm{~Hz}$, $-\mathrm{OH}), 4.19-4.39$ (2 H, m, -O-C $\left.\underline{\mathrm{H}}_{2}^{-}\right), 4.39-4.64(2 \mathrm{H}$, m, $\left.-\mathrm{CH}(\mathrm{OH})-,-\mathrm{O}-\mathrm{CH}_{2}-\mathrm{C} \underline{\mathrm{H}}-\mathrm{CH}\left(\mathrm{CH}_{3}\right)_{2}\right), 5.48$ (1 H, ddt, $J=15.4,6.5,1.36 \mathrm{~Hz},-\mathrm{CH}=\mathrm{CH}-), 5.69(1 \mathrm{H}, \mathrm{d}, J=5.1 \mathrm{~Hz}$, -CO-CH(Br)-), 5.87 (1 H, dt, J=15.4, 6.8 Hz, -C $\underline{\mathrm{H}}=\mathrm{CH}-$ ).

(S)-3-((2R,3R,E)-2-Azido-3-hydroxyoctadec4-enoyl)-4-isopropyloxazolidin-2-one (11): A solution of $10(7.87 \mathrm{~g}, 16.1 \mathrm{mmol})$ and $\mathrm{NaN}_{3}(2.11 \mathrm{~g}$, $32.5 \mathrm{mmol})$ in DMSO $(41 \mathrm{~mL})$ was stirred at room temperature for $1.5 \mathrm{~h}$. The reaction was quenched with water and extracted with AcOEt. The organic layer was washed with water and brine, dried over $\mathrm{MgSO}_{4}$, and evaporated. The residue was flash-chromatographed on silica gel (200 g, hexane/AcOEt, $4: 1)$ to give compound 11 (6.22 g, 86\%). $11{ }^{1} \mathrm{H}$ NMR $\delta 0.82-0.97$ (9 $\left.\mathrm{H}, \mathrm{m},-\mathrm{CH}_{3},-\mathrm{CH}\left(\mathrm{CH}_{3}\right)_{2}\right), 1.16-1.45\left(22 \mathrm{H}, \mathrm{m},-\mathrm{CH}_{2}-\right)$, $2.08\left(2 \mathrm{H}, \mathrm{q}, \mathrm{J}=6.86 \mathrm{~Hz},-\mathrm{CH}=\mathrm{CH}-\underline{\mathrm{H}}_{2}-\right), 2.27-2.51(2$ $\left.\mathrm{H}, \mathrm{m},-\mathrm{CH}\left(\mathrm{CH}_{3}\right)_{2},-\mathrm{OH}\right), 4.22-4.32\left(1 \mathrm{H}, \mathrm{m},-\mathrm{O}-\mathrm{CH}_{2}-\mathrm{C} \underline{\mathrm{H}}-\right.$ $\left.\mathrm{CH}\left(\mathrm{CH}_{3}\right)_{2}\right), 4.32-4.40$ (1 H, m, -O- $\left.\underline{\mathrm{CH}}_{2} \underline{2}^{-}\right), 4.40-4.55$ (2 H, m, - $\left.\mathrm{CH}(\mathrm{OH})-,-\mathrm{O}-\mathrm{C}_{2} \underline{2}^{-}\right), 5.10(1 \mathrm{H}, \mathrm{d}, J=7.7 \mathrm{~Hz}$, $\left.-\mathrm{CH}\left(\mathrm{N}_{3}\right)-\right), 5.60$ (1 H, ddt, $J=15.4,7.1,1.3 \mathrm{~Hz},-\mathrm{CH}=\mathrm{CH}-$ ), 5.89 ( $1 \mathrm{H}, \mathrm{dt}, \mathrm{J}=15.4,6.6 \mathrm{~Hz},-\mathrm{CH}=\mathrm{CH}-$ ).
(S)-3-((2R,3R,E)-2-Azido-3-((tert-butyldimethylsilyl)oxy)octadec-4-enoyl)-4-isopropyloxazolidin2-one (12): To a solution of 11 (6.22 g, $13.8 \mathrm{mmol})$ in THF $(69 \mathrm{~mL})$ at $0^{\circ} \mathrm{C}$ was added 2,6-lutidine $(3.2 \mathrm{~mL}$, $27.6 \mathrm{mmol}$ ) followed by tert-butyldimethylsilyltriflate (4.8 mL, $20.9 \mathrm{mmol})$. The mixture was stirred for $30 \mathrm{~min}$ at $0^{\circ} \mathrm{C}$ and then at room temperature for $1 \mathrm{~h}$. The reaction was quenched with water and extracted with AcOEt. The organic layer was washed with water and brine, dried over $\mathrm{MgSO}_{4}$, and evaporated. The residue was flash-chromatographed on silica gel (200 g, hexane/AcOEt, $15: 1)$ to give compound 12 (6.21 g, 80\%). $12{ }^{1} \mathrm{H}$ NMR $\delta 0.05\left(3 \mathrm{H}, \mathrm{s},-\mathrm{Si}\left(\mathrm{CH}_{3}\right)_{2}\left(\mathrm{CH}_{3}\right)_{3}\right), 0.08(3 \mathrm{H}, \mathrm{s}$, $\left.-\mathrm{Si}\left(\mathrm{C}_{\underline{3}}\right)_{2}\left(\mathrm{CH}_{3}\right)_{3}\right), 0.82-0.90\left(15 \mathrm{H}, \mathrm{m},-\mathrm{Si}\left(\mathrm{CH}_{3}\right)_{2}\left(\mathrm{CH}_{3}\right)_{3}\right.$, $\left.-\mathrm{CH}\left(\underline{\mathrm{CH}}_{3}\right)_{2}\right), 0.91-0.96$ (3 H, m, $\left.-\underline{\mathrm{CH}}_{3}\right), 1.16-1.43(22$ $\left.\mathrm{H}, \mathrm{m},-\underline{\mathrm{CH}}_{2^{-}}\right), 2.01-2.08$ (2 H, m, $-\mathrm{CH}=\mathrm{CH}-\underline{\mathrm{C}}_{2^{-}}$), 2.252.35 (1 H, m, - $\left.\mathrm{C} \underline{\mathrm{H}}\left(\mathrm{CH}_{3}\right)_{2}\right), 4.22-4.26\left(1 \mathrm{H}, \mathrm{m},-\mathrm{O}-\mathrm{CH}_{2}-\right)$, 4.29-4.35 (1 H, m, - $\left.-\mathrm{CH}_{2}-\underline{H}^{-}\right) 4.49$ (1 H, dt, J=8.1, $\left.3.4 \mathrm{~Hz},-\mathrm{O}-\mathrm{CH}_{2}-\mathrm{CH}-\mathrm{CH}\left(\mathrm{CH}_{3}\right)_{2}\right), 4.64(1 \mathrm{H}, \mathrm{dd}, \mathrm{J}=8.0$, $\left.6.6 \mathrm{~Hz},-\mathrm{CH}\left(\mathrm{N}_{3}\right)-\mathrm{CH}-\right), 5.21\left(1 \mathrm{H}, \mathrm{d}, J=6.4 \mathrm{~Hz},-\mathrm{CH}\left(\mathrm{N}_{3}\right)-\right)$, 5.52 ( $1 \mathrm{H}, \mathrm{dd}, J=15.3,8.1 \mathrm{~Hz},-\mathrm{CH}=\mathrm{C} \underline{\mathrm{H}}-), 5.75$ (1 H, dt, $J=15.2,6.8 \mathrm{~Hz},-\mathrm{CH}=\mathrm{CH}-)$.

(2S,3R,E)-2-Azido-3-((tert-butyldimethylsilyl) oxy)octadec-4-en-1-ol (13): To a solution of 12 $(6.21 \mathrm{~g}, 11.0 \mathrm{mmol})$ in THF $(55 \mathrm{~mL})$ at $0^{\circ} \mathrm{C}$ was added $\mathrm{LiBH}_{4}(0.722 \mathrm{~g}, 33.1 \mathrm{mmol})$. The mixture was stirred at $0^{\circ} \mathrm{C}$ for $1.5 \mathrm{~h}$ and then at room temperature for $30 \mathrm{~min}$. The mixture was cooled to $0^{\circ} \mathrm{C}$ and then diluted with AcOEt. The reaction was quenched with saturated $\mathrm{NH}_{4} \mathrm{Cl}$ aq. and extracted with AcOEt. The organic layer was washed with water and brine, dried over $\mathrm{MgSO}_{4}$, and evaporated. The residue was chromatographed on silica gel (100 g, hexane/AcOEt, $15: 1)$ to give compound 13 (4.18 g, 86\%). $13{ }^{1} \mathrm{H}$ NMR $\delta 0.05$ (3 H, s, $\left.-\mathrm{Si}\left(\mathrm{CH}_{3}\right)_{2}\left(\mathrm{CH}_{3}\right)_{3}\right), 0.09\left(3 \mathrm{H}, \mathrm{s},-\mathrm{Si}\left(\mathrm{C}_{\underline{3}}\right)_{2}\left(\mathrm{CH}_{3}\right)_{3}\right)$, 0.82-0.97 (12 H, m, $\left.-\mathrm{Si}\left(\mathrm{CH}_{3}\right)_{2}\left(\mathrm{CH}_{3}\right)_{3},-\mathrm{CH}_{3}\right), 1.15-1.44$ $\left(22 \mathrm{H}, \mathrm{m},-\mathrm{CH}_{2} \underline{2}^{-}, 1.95-2.21\left(3 \mathrm{H}, \mathrm{m},-\mathrm{CH}=\mathrm{CH}-\mathrm{CH}_{2} \underline{2}^{-}\right.\right.$, $-\mathrm{OH}), 3.36-3.45$ (1 H, m, $\left.-\mathrm{CH}\left(\mathrm{N}_{3}\right)-\right), 3.62-3.83$ (2 $\left.\mathrm{H}, \mathrm{m}, \mathrm{HO}-\mathrm{CH}_{2}-\right)$, 4.22 (1 H, dd, J=7.2, $5.3 \mathrm{~Hz},-\mathrm{CH}-\mathrm{O}-$ $\left.\mathrm{Si}\left(\mathrm{CH}_{3}\right)_{2-}\right), 5.46$ (1 H, ddt, J=15.4, 7.4, $1.3 \mathrm{~Hz}$, -CH=C吕-), 5.70 (1 H, dt, J=15.4, 6.7 Hz, -CH=CH-).

(2S,3R,E)-2-Amino-3-((tert-butyldimethylsilyl) oxy)octadec-4-en-1-ol (14): A solution of 13 (4.18 g, $9.51 \mathrm{mmol})$ in THF $(120 \mathrm{~mL})$ was diluted with water (15 mL). To this solution, triphenylphosphine (4.99 g, $19.0 \mathrm{mmol}$ ) was added and the mixture was stirred at $60^{\circ} \mathrm{C}$ for $15 \mathrm{~h}$. After evaporation, the residue was extracted with water and $\mathrm{CH}_{2} \mathrm{Cl}_{2}$. The organic layer was washed with water and brine, dried over $\mathrm{MgSO}_{4}$, and evaporated. The residue was flash-chromatographed on silica gel $\left(200 \mathrm{~g}, \mathrm{CH}_{2} \mathrm{Cl}_{2} / \mathrm{MeOH}, 40: 1\right.$ and then $1: 1$ ) to give compound $\mathbf{1 4}(3.24 \mathrm{~g}, 82 \%) .14^{1} \mathrm{H}$ NMR $\delta 0.03$ (3 H, s, $\left.-\mathrm{Si}\left(\mathrm{CH}_{3}\right)_{2}\left(\mathrm{CH}_{3}\right)_{3}\right), 0.07$ (3 H, s, $\left.-\mathrm{Si}\left(\mathrm{C}_{\underline{3}}\right)_{2}\left(\mathrm{CH}_{3}\right)_{3}\right)$, $0.70-0.98\left(12 \mathrm{H}, \mathrm{m},-\mathrm{Si}\left(\mathrm{CH}_{3}\right)_{2}\left(\mathrm{CH}_{3}\right)_{3},-\underline{\mathrm{CH}}_{3}\right), 1.13-$ $1.50\left(22 \mathrm{H}, \mathrm{m},-\mathrm{CH}_{2^{-}}\right), 1.76(3 \mathrm{H}$, broad s), $2.05(2 \mathrm{H}$, q, $\left.J=6.8 \mathrm{~Hz},-\mathrm{CH}=\mathrm{CH}-\underline{\mathrm{H}}_{2^{-}}\right), 2.75(1 \mathrm{H}, \mathrm{q}, J=5.3 \mathrm{~Hz}$, -CH$\left.\left(\mathrm{NH}_{2}\right)-\right), 3.53-3.68$ (2 H, m, HO-C $\left.\underline{\mathrm{H}}_{2}{ }^{-}\right), 4.01(1 \mathrm{H}$, $\left.\mathrm{t}, J=6.3 \mathrm{~Hz},-\mathrm{CH}-\mathrm{O}-\mathrm{Si}\left(\mathrm{CH}_{3}\right)_{2}-\right), 5.38(1 \mathrm{H}, \mathrm{dd}, J=15.4$, $7.8 \mathrm{~Hz},-\mathrm{CH}=\mathrm{CH}-$ ), 5.65 (1 H, dt, $J=15.3,6.8 \mathrm{~Hz}$, $-\mathrm{CH}=\mathrm{CH}-$ ). 

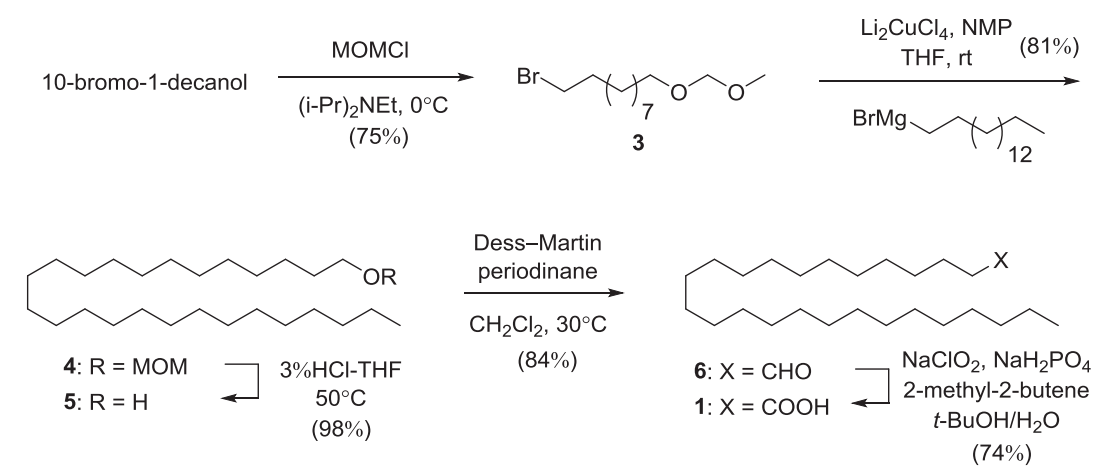

Scheme 1. Synthetic scheme of hexacosanoic acid (1).

N-((2S,3R,E)-3-((tert-Butyldimethylsilyl)oxy)1-hydroxyoctadec-4-en-2-yl)hexacosanamide (15): To a solution of $\mathbf{1 4}(0.207 \mathrm{~g}, 0.500 \mathrm{mmol})$ and $\mathbf{1}$ $(0.196 \mathrm{~g}, 0.495 \mathrm{mmol})$ in $\mathrm{CH}_{2} \mathrm{Cl}_{2}(55 \mathrm{~mL})$ was added EEDQ $(0.183 \mathrm{~g}, 0.738 \mathrm{mmol})$. The mixture was stirred at $35^{\circ} \mathrm{C}$ for $10 \mathrm{~h}$. After evaporation the residue was flash chromatographed on silica gel $(80 \mathrm{~g}$, hexane/ ether, $3: 1$ and then $\mathrm{CH}_{2} \mathrm{Cl}_{2}$ /ether, $\left.1: 1\right)$ to give compound $15(0.335 \mathrm{~g}, 84 \%) .15{ }^{1} \mathrm{H}$ NMR $\delta 0.03(3 \mathrm{H}$, s, $\left.-\mathrm{Si}\left(\mathrm{CH}_{3}\right)_{2}\left(\mathrm{CH}_{3}\right)_{3}\right), 0.06\left(3 \mathrm{H}, \mathrm{s},-\mathrm{Si}\left(\mathrm{CH}_{3}\right)_{2}\left(\mathrm{CH}_{3}\right)_{3}\right), 0.63-$ $0.98\left(15 \mathrm{H}, \mathrm{m},-\mathrm{Si}\left(\mathrm{CH}_{3}\right)_{2}\left(\mathrm{CH}_{3}\right)_{3},-\left(\mathrm{CH}_{2}\right)_{12}-\mathrm{CH}_{3},-\left(\mathrm{CH}_{2}\right)_{24}-\right.$ $\left.\mathrm{CH}_{3}\right), 0.98-1.15\left(66 \mathrm{H}, \mathrm{m},-\mathrm{CH}_{2^{-}}\right), 1.57-1.70(2 \mathrm{H}, \mathrm{m}$, $\left.-\mathrm{NH}-\mathrm{CO}-\mathrm{CH}_{2}-\mathrm{CH}_{2}-\right), 2.05(2 \mathrm{H}, \mathrm{q}, \mathrm{J}=6.8 \mathrm{~Hz},-\mathrm{CH}=\mathrm{CH}-$ $\left.\mathrm{CH}_{2} \underline{2}^{-}\right), 2.21\left(2 \mathrm{H}, \mathrm{t}, J=7.5 \mathrm{~Hz},-\mathrm{NH}-\mathrm{CO}-\mathrm{CH}_{2} \underline{2}^{-}\right), 3.25(1 \mathrm{H}$, dd, $J=10.0,1.7 \mathrm{~Hz}, \underline{\mathrm{HO}}-)$, 3.55 ( $1 \mathrm{H}, \mathrm{td}, J=10.7,3.1 \mathrm{~Hz}$, HO-C $\left.\underline{H}_{2}{ }^{-}\right), 3.74-3.86$ (1 H, m, - $\left.\mathrm{CH}-\mathrm{O}-\mathrm{Si}\left(\mathrm{CH}_{3}\right)_{2}-\right)$, 4.04 (1 $\left.\mathrm{H}, \mathrm{dt}, J=11.9,2.28 \mathrm{~Hz}, \mathrm{HO}-\mathrm{CH}_{2}-\right), 4.38-4.49$ (1 H, m, -Cㅡ(HCO)-), 5.46 (1 H, dd, J=15.5, $6.2 \mathrm{~Hz},-\mathrm{CH}=\mathrm{C} \underline{\mathrm{H}}-)$, 5.72 ( $1 \mathrm{H}, \mathrm{dt}, J=15.5,6.7 \mathrm{~Hz},-\mathrm{CH}=\mathrm{CH}-), 6.25$ ( $1 \mathrm{H}, \mathrm{d}$, $J=7.5 \mathrm{~Hz},-\mathrm{N} \underline{\mathrm{H}}-\mathrm{CO}-) .{ }^{13} \mathrm{C} \mathrm{NMR} \delta-4.9,-4.2,14.3(2$ carbons), 18.2, 22.9 (2 carbons), 25.8, 26.0 (4 carbons), 29.29, 29.35, 29.6 (3 carbons), 29.65, 29.67, 29.8 (3 carbons), 29.85 (5 carbons), 29.89 (13 carbons), 32.1 (2 carbons), 32.4, 37.0, 54.6, 62.5, 76.2, 130, 133.8, 173.3.

N-((2S,3R,E)-1,3-Dihydroxyoctadec-4-en-2-yl) hexacosanamide (2): To a solution of $15(0.137 \mathrm{~g}$, $0.173 \mathrm{mmol})$ in THF $(20 \mathrm{~mL})$ was added acetic acid (15 $\mu \mathrm{L}, 0.262 \mathrm{mmol}$ ) and TBAF (1.0 M solution in THF, $260 \mu \mathrm{L}, 0.260 \mathrm{mmol})$. The mixture was stirred at $35^{\circ} \mathrm{C}$ for $5 \mathrm{~h}$. After evaporation the residue was diluted with water and extracted with $\mathrm{CH}_{2} \mathrm{Cl}_{2}$. The organic layer was washed with water and brine, dried over $\mathrm{MgSO}_{4}$, and evaporated. The residue was flash-chromatographed on silica gel $\left(50 \mathrm{~g}, \mathrm{CHCl}_{3} / \mathrm{MeOH}, 70: 1\right)$ to give compound $2(0.112 \mathrm{~g}, 96 \%) .2{ }^{1} \mathrm{H}$ NMR $\delta 0.88(6 \mathrm{H}, \mathrm{t}, J=6.6 \mathrm{~Hz}$, $\left.-\left(\mathrm{CH}_{2}\right)_{12}-\mathrm{CH}_{3},-\left(\mathrm{CH}_{2}\right)_{24}-\underline{\mathrm{CH}}_{3}\right), 0.99-1.52\left(66 \mathrm{H}, \mathrm{m},-\mathrm{CH}_{2^{-}}\right)$, 1.63-1.72 (2 H, m, -NH-CO-CH $\left.-\mathrm{CH}_{2}-\right), 2.06(2 \mathrm{H}, \mathrm{q}$, $\left.J=6.8 \mathrm{~Hz},-\mathrm{CH}=\mathrm{CH}-\mathrm{CH}_{2}{ }^{-}\right), 2.23(2 \mathrm{H}, \mathrm{t}, J=7.3 \mathrm{~Hz},-\mathrm{NH}-$ CO-C $\left.\underline{H}_{2}{ }^{-}\right), 2.63-2.82$ (1 H, m, - $\left.\mathrm{CH}(\mathrm{NHCO})-\mathrm{CH}(\mathrm{OH})-\right)$,

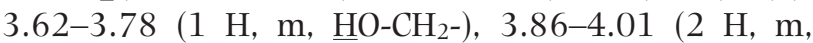
HO-C $\left.\underline{\mathrm{H}}_{2}{ }^{-}\right), 4.25-4.38$ (1 H, m, -C $\left.\underline{\mathrm{H}}(\mathrm{NHCO})-\right), 5.53$ (1 H, dd, $J=15.5,6.4 \mathrm{~Hz},-\mathrm{CH}=\mathrm{C} \underline{\mathrm{H}}-), 5.79$ (1 H, dt, $J=14.9$, $7.1 \mathrm{~Hz},-\mathrm{CH}=\mathrm{CH}-), 6.24$ (1 H, d, J=7.3 Hz, -N $\underline{H}-\mathrm{CO}-$ ). ${ }^{13} \mathrm{C}$ NMR $\delta 14.3$ (2 carbons), 22.9 (2 carbons), 26.0,
29.3, 29.45, 29.51, 29.6 (3 carbons), 29.71, 29.74, 29.85, 29.88 (4 carbons), 29.9 (16 carbons), 32.2 (2 carbons), 32.5, 37.1, 54.7, 62.7, 74.9, 129.0, 134.5, 174.2. HRMS (ESI ${ }^{-}$) calcd. for ${ }^{12} \mathrm{C}_{44}{ }^{1} \mathrm{H}_{87}{ }^{35} \mathrm{Cl}_{1}{ }^{14} \mathrm{~N}_{1}{ }^{16} \mathrm{O}_{3}$ 712.6375 , found 712.6354 .

\section{RESULTS AND DISCUSSION}

\section{Synthesis of hexacosanoic acid}

The synthetic scheme is shown in Scheme 1. To develop an efficient method applicable to the chemical synthesis of various carbon-chain fatty acids, we planned the synthesis of hexacosanoic acid (C26:0) (1) by applying the coupling of two alkyl units as a key step. As shown in Scheme 1, if C-C bond formation occurs between C10-unit compound 3 and C16-unit, it would be an efficient synthetic scheme for a variety of VLCFAs. 10-Bromo-1-decanol was protected by using chloromethyl methyl ether to provide methoxymethyl ether 3 . The cross coupling reaction between compound $\mathbf{3}$ and hexadecyl magnesium bromide catalyzed by $\mathrm{Li}_{2} \mathrm{CuCl}_{4}$ yielded hexacosanol derivative $\mathbf{4}$ in a moderate yield $(58 \%)(7,8)$. Cahiez et al. reported the addition of $\mathrm{N}$-methylpyrrolidone improved $\mathrm{Cu}$-catalyzed cross coupling (9). When we applied this method, the yield of compound $\mathbf{4}$ was improved to $81 \%$. Compound $\mathbf{4}$ was deprotected to provide hexacosanol $\mathbf{5}$ in $98 \%$ yield.

Hexacosanol $\mathbf{5}$ has a very long chain of carbon hydride, which possesses specific physical properties in organic synthesis. It is a white waxy solid at room temperature and scarcely soluble in organic solvents as well as water. It seems that only $\mathrm{CH}_{2} \mathrm{Cl}_{2}$ and chloroform are slightly soluble solvents, indicating that the property makes organic reactions difficult. It was not exceptional for the oxidation reaction of primary alcohol $\mathbf{5}$ to carboxylic acid 1. First, one-step oxidation of hexacosanol 5 to hexacosanoic acid (1) was tried by using several oxidation reagents such as bismuth (III) oxide (10), but the reaction did not proceed at all. Therefore, we changed one-step oxidation to two steps via aldehyde. First we tried Swern oxidation of alcohol $\mathbf{5}$ to aldehyde $\mathbf{6}$, but we failed because alcohol $\mathbf{5}$ didn't dissolve in $\mathrm{CH}_{2} \mathrm{Cl}_{2}$ at $-78^{\circ} \mathrm{C}$. Next we tried oxidation using DessMartin periodinane (11), and we succeeded in obtaining 6 in $84 \%$ yield when the reaction in $\mathrm{CH}_{2} \mathrm{Cl}_{2}$ was carried out at $30^{\circ} \mathrm{C}$. At that temperature alcohol $\mathbf{5}$ was some- 

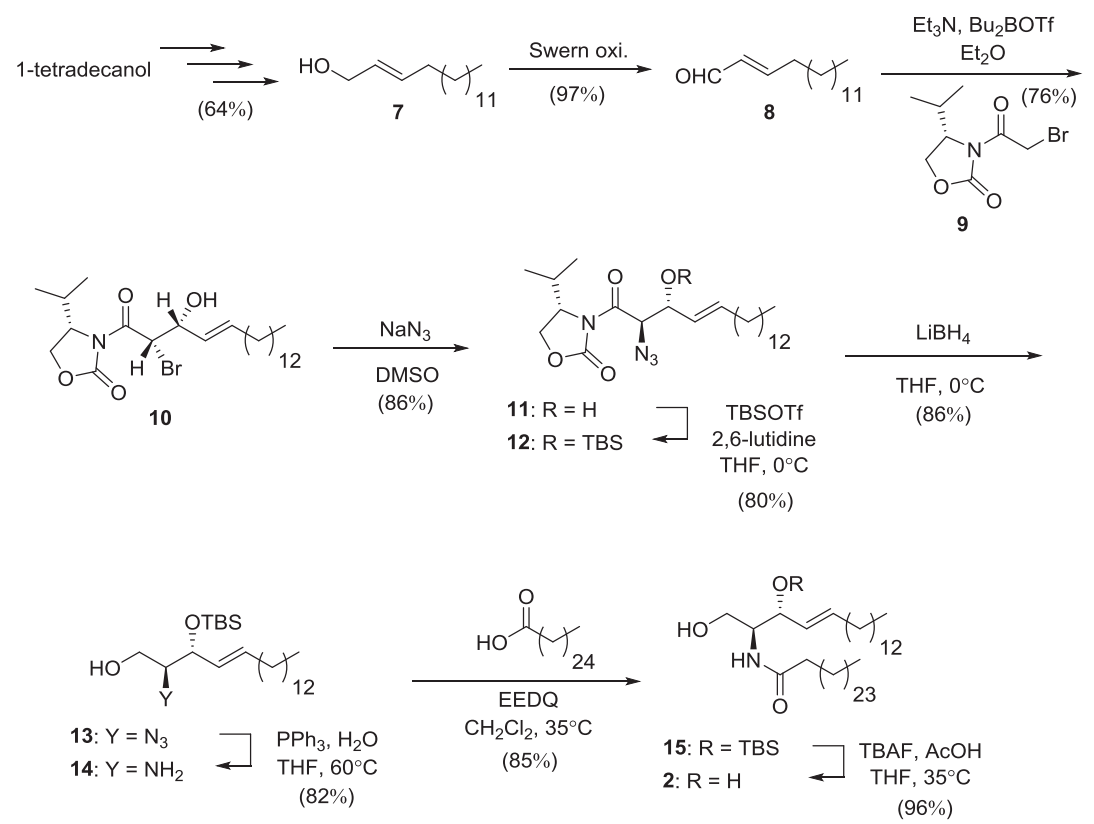

Scheme 2. Synthetic scheme of the ceramide $\mathbf{2}$ which contains hexacosanoic acid.

how soluble in $\mathrm{CH}_{2} \mathrm{Cl}_{2}$. Finally, oxidation of $\mathbf{6}$ to hexacosanoic acid (1) was performed by Pinnick oxidation (12) in 74\% yield. Aldehyde $\mathbf{6}$ was also hard to dissolve in organic solvents but it was a little more soluble than alcohol 5. This is the first report of an efficient chemical synthesis of hexacosanoic acid (C26:0) (1) by carboncarbon coupling reaction.

Synthesis of ceramide containing hexacosanoic acid

The synthetic scheme is shown in Scheme 2. According to the reported procedure, we obtained allylic alcohol 7 from 1-tetradecanol in three steps in yield of $64 \%$ $(13,14)$. Alcohol 7 was converted to aldehyde 8 by Swern oxidation in $97 \%$ yield. To generate two desired stereocenters together with two-carbon elongation, we prepared $(+)-(4 S)$-3-bromoacetyl-4-(1-methlethyl)2-oxazolidinone 9 (15) which was then coupled with aldehyde $\mathbf{8}$ to give two-carbon elongated compound 10 in 76\% yield (16). Oxazolidinone 9 was used as a chiral auxiliary for the diastereoselective aldol reaction. This reaction including the reaction mechanism and the stereochemistry of products has been reported in detail $(15,16)$. Compound $\mathbf{1 0}$ was treated with $\mathrm{NaN}_{3}$ to give azide 11, whose secondary alcohol was then protected to give compound 12. The amide group of $\mathbf{1 2}$ was reduced with $\mathrm{LiBH}_{4}$ to give alcohol 13 (59\% yield from 10) (16). The azide group of 13 was reduced by the Staudinger reaction to give amine $\mathbf{1 4}$ in $82 \%$ yield $(17,18)$. Spectral data of known compounds 9-14 were identical with those reported.

Compound 14 and hexacosanoic acid (C26:0) (1) were treated with $\mathrm{N}$-ethoxycarbonyl-2-ethoxy-1,2-dihydroquinoline as a condensation reagent in $\mathrm{CH}_{2} \mathrm{Cl}_{2}$ at $35^{\circ} \mathrm{C}$ for $10 \mathrm{~h}$ to provide condensation product 15 in $85 \%$ yield (19-21). The reaction required $10 \mathrm{~h}$ because hexacosanoic acid (1) was scarcely soluble in the solvent. Compound $\mathbf{1 5}$ was deprotected by treatment with tetra- $n$-butylammonium fluoride to give ceramide $\mathbf{2}$ in yield of only $7 \%$, but an additon of one equivalent acetic acid to the reaction dramatically improved the yield of ceramide $\mathbf{2}$ to $96 \%$ (22). Ceramide $\mathbf{2}$ was hard to dissolve in organic solvents but it was a little soluble in ethanol and dimethylsulfoxide. This is the first report of the chemical synthesis of the ceramide containing hexacosanoic acid (1).

\section{CONCLUSION}

We synthesized hexacosanoic acid (C26:0) (1) and the ceramide $\mathbf{2}$ containing 1. Combination of synthetic Schemes 1 and 2 allows obtaining various ceramides containing a motif of poorly soluble VLCFA. These compounds are useful for investigation of details of these compounds related to diseases.

\section{Acknowledgments}

A part of this work was supported by the Platform for Drug Discovery, Informatics, and Structural Life Science and by a Grant-in-Aid for Scientific Research (no. 22790116 and 25860090) from the Ministry of Education, Culture, Sports, Science and Technology, Japan. We also thank the MEXT-Supported Program for the Strategic Research Foundation at Private Universities (20132017) and the Takeda Science Foundation, Japan, for financial support.

\section{REFERENCES}

1) Tsuji S, Sano T, Ariga T, Miyatake T. 1981. Increased synthesis of hexacosanoic acid (C26:0) by cultured skin fibroblasts from patients with adrenoleukodystrophy (ALD) and adrenomyeloneuropathy (AMN). J Biochem 90: $1233-1236$.

2) Koike R, Tsuji S, Ohno T, Suzuki Y, Orii T, Miyatake T. 1991. Physiological significance of fatty acid elongation system in adrenoleukodystrophy. J Neurol Sci 103: 188-194.

3) Antoku Y, Tsukamoto K, Miyoshi Y, Nagino H, Anezaki 
M, Suwa K, Narabe Y. 2000. Correlations of elevated levels of hexacosanoate in erythrocyte membranes with risk factors for atherosclerosis. Atherosclerosis 153: 169-173.

4) Miyazaki T, Shimada K, Hiki M, Kume A, Kitamura Y, Oshida K, Yanagisawa N, Kiyanagi T, Matsumori R, Daida H. 2014. High hexacosanoic acid levels are associated with coronary artery disease. Atherosclerosis 233: 429-433.

5) Molano A, Huang Z, Marko MG, Azzi A, Wu D, Wang E, Kelly SL, Merrill Jr AH, Bunnell SC, Meydani SN. 2012. Age-dependent changes in the sphingolipid composition of mouse CD4 + T cell membranes and immune synapses implicate glucosylceramides in age-related $\mathrm{T}$ cell dysfunction. PLoS One 7: e47650.

6) Kume A, Miyazaki T, Kitamura Y, Oshida K, Yanagisawab N, Takizawa H, Fujii K, Kiyanagi T, Sumiyoshi K, Ohmura H, Mokuno H, Shimada K, Daida H. 2008. High levels of saturated very long-chain fatty acid (hexacosanoic acid; C26:0) in whole blood are associated with metabolic syndrome in Japanese men. Diabetes Res Clin Pract 80: 259-264.

7) Kulkarni SM, Mamdapur VR, Chadha MS. 1983. A simple synthesis of 1-triacontanol. Indian J Chem Sect B: Org Chem Incl Med Chem 22B: 683.

8) Lin H-S, Paquette LA. 1994. A convenient method for determining the concentration of grignard reagents. Synth Commun 24: 2503-2506.

9) Cahiez G, Chaboche C, Jézéquel M. 2000. Cu-catalyzed alkylation of grignard reagents: A new efficient procedure. Tetrahedron 56: 2733-2737.

10) Malik P, Chakraborty D. 2010. Bismuth (III) oxide catalyzed oxidation of alcohols with tert-butyl hydroperoxide. Synthesis 21: 3736-3740.

11) Iwayama $Y$, Ando H, Ishida H, Kiso M. 2009. A first total synthesis of ganglioside HLG-2. Chem-Eur J 15: 4637-4648.

12) Bal BS, Childers WE Jr, Pinnick HW. 1981. Oxidation of $\alpha, \beta$-unsaturated aldehydes. Tetrahedron 37: 2091-2096.
13) Yadav JS, Raghavendra Rao KV, Ravindar K, Subba Reddy BV. 2010. Total synthesis of (+)-aculeatin D and (+)-6-epi-aculeatin D. Synlett 1: 51-54.

14) Sabitha G, Prasad MN, Shankaraiah K, Reddy NM, Yadav JS. 2010. Convergent synthesis of passifloricin A via a prins cyclisation and olefin cross-metathesis approach. Synthesis 22: 3891-3898.

15) Abdel-Magid A, Pridgen LN, Eggleston DS, Lantos I. 1986. Metal-assisted aldol condensation of chiral $\alpha$-halogenated imide enolates: A stereocontrolled chiral epoxide synthesis. J Am Chem Soc 108: 4595-4602.

16) Nicolaou KC, Caulfield TJ, Katoaka H. 1990. Total synthesis of globotriaosylceramide (Gb3) and lysoglobotriaosylceramide (lysoGb3). Carbohydr Res 202: 177-191.

17) Dong Z, Butcher JA Jr. 1991. A useful synthesis of D-erythro-sphingomyelins. Tetrahedron Lett 32: 5291-5294.

18) Tarnowski A, Retz O, Bär T, Schmidt RR. 2005. Synthesis of sphingosine-1-phosphonate and homosphingosine1-phosphonate. Eur J Org Chem 2005: 1129-1141.

19) Romine JL, Laurent DRS, Leet JE, Martin SW, SerranoWu MH, Yang F, Gao M, Donald R O'Boyle II, Lemm JA, Sun J-H, Nower PT, Huang XS, Deshpande M, Meanwell NA, Snyder LB. 2011. Inhibitors of HCV NS5A: From iminothiazolidinones to symmetrical stilbenes. ACS Med Chem Lett 2: 224-229.

20) Hyun MH, Kang MH, Han SC. 1999. Use of 2-ethoxy1-(ethoxycarbonyl)-1,2-dihydroquinoline as a convenient reagent for the selective protection or derivatization of 2-hydroxycarboxylic acids. Tetrahedron Lett 40: 3435-3438.

21) Zacharie B, Connolly TP, Penney CL. 1995. A simple one-step conversion of carboxylic acids to esters using EEDQ. J Org Chem 60: 7072-7074.

22) Smith III AB, Condon SM, McCauley JA, Leazer Jr JL, Leahy JW, Maleczka Jr RE. 1997. A unified total synthesis of the immunomodulators (-)-rapamycin and (-)-27-demethoxyrapamycin: Assembly of the common $\mathrm{C}(1-20)$ perimeter and final elaboration. J Am Chem Soc 119: $962-973$. 